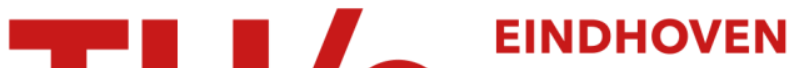 \\ UNIVERSITY OF \\ TECHNOLOGY
}

\section{Sharp asymptotics for stochastic dynamics with parallel updating rule}

Citation for published version (APA):

Nardi, F. R., \& Spitoni, C. (2012). Sharp asymptotics for stochastic dynamics with parallel updating rule. (Report Eurandom; Vol. 2012001). Eurandom.

Document status and date:

Published: 01/01/2012

\section{Document Version:}

Publisher's PDF, also known as Version of Record (includes final page, issue and volume numbers)

\section{Please check the document version of this publication:}

- A submitted manuscript is the version of the article upon submission and before peer-review. There can be important differences between the submitted version and the official published version of record. People interested in the research are advised to contact the author for the final version of the publication, or visit the $\mathrm{DOI}$ to the publisher's website.

- The final author version and the galley proof are versions of the publication after peer review.

- The final published version features the final layout of the paper including the volume, issue and page numbers.

Link to publication

\section{General rights}

Copyright and moral rights for the publications made accessible in the public portal are retained by the authors and/or other copyright owners and it is a condition of accessing publications that users recognise and abide by the legal requirements associated with these rights.

- Users may download and print one copy of any publication from the public portal for the purpose of private study or research.

- You may not further distribute the material or use it for any profit-making activity or commercial gain

- You may freely distribute the URL identifying the publication in the public portal.

If the publication is distributed under the terms of Article 25fa of the Dutch Copyright Act, indicated by the "Taverne" license above, please follow below link for the End User Agreement:

www.tue.nl/taverne

Take down policy

If you believe that this document breaches copyright please contact us at:

openaccess@tue.nl

providing details and we will investigate your claim. 
EURANDOM PREPRINT SERIES

2012-001

January 24, 2012

Sharp Asymptotics for Stochastic Dynamics with Parallel Updating Rule

F.R. Nardi, C. Spitoni

ISSN 1389-2355 


\title{
Sharp Asymptotics for Stochastic Dynamics with Parallel Updating Rule
}

\author{
F. R. Nardi ${ }^{12}$ \\ C. Spitoni ${ }^{3}$
}

January 25, 2012

\begin{abstract}
In this paper we study the metastability problem for a stochastic dynamics with a parallel updating rule; in particular we consider a finite volume Probabilistic Cellular Automaton (PCA) in a small external field at low temperature regime. We are interested in the nucleation of the system, i.e., the typical excursion from the metastable phase (the configuration with all minuses) to the stable phase (the configuration with all pluses), triggered by the formation of a critical droplet. The main result of the paper is the sharp estimate of the nucleation time: we show that the nucleation time divided by its average converges to an exponential random variable and that the rate of the exponential random variable is an exponential function of the inverse temperature $\beta$ times a prefactor that does not scale with $\beta$. Our approach combines geometric and potential theoretic arguments.
\end{abstract}

MSC2000. 60K35, 82C26.

Key words and phrases. Stochastic dynamics; probabilistic cellular automata; metastability; potential theory; Dirichlet form; capacity

Acknowledgment. The authors acknowledge A. Bovier and E. Cirillo for useful discussion and suggestions.

\section{Introduction}

Metastable states are very common in nature and are typical of systems close to a first order phase transition. It is often observed that a system can persist for a long period of time in a phase not favored by the thermodynamic parameters; classical examples are the super-saturated vapor and the magnetic hysteresis. The rigorous description of this

\footnotetext{
${ }^{1}$ EURANDOM, P.O. Box 513, 5600 MB Eindhoven, The Netherlands

${ }^{2}$ Department of Mathematics and Computer Science Eindhoven University of Technology, P.O. Box 513, 5600 MB Eindhoven, The Netherlands

${ }^{3}$ Department of Medical Statistics and Bioinformatics, Leiden University Medical Centre , P.O. Box 9600, 2300 RC Leiden, The Netherlands
} 
phenomenon in the framework of well defined mathematical models is relatively recent, dating back to the pioneering paper [CGOV], and has experienced substantial progress in the last decade. See $[\mathrm{OV}],[\mathrm{Bo}],[\mathrm{Ho}]$ for reviews and for a list of the most important papers on this subject.

A natural setup in which the phenomenon of metastability can be studied is that of Markov chains, or Markov processes, describing the time evolution of a statistical mechanical system. Think for instance to a stochastic lattice spin system. In this context powerful theories (see [BEGK], [OS], [OS2] and [OV]) have been developed. Furthermore, the results in [MNOS] improved those in [OS] in the direction of minimizing the number of model-dependent inputs necessary to describe the metastable behavior of the system.

Whatever approach is chosen, a key model dependent question is the computation of the minimal energy barrier, called communication energy, to be overcome by a path connecting the metastable to the stable state. Such a problem is in general quite complicated and becomes particularly difficult when the dynamics has a parallel character: as soon simultaneous updates are allowed, the continuity constraint on the structure of the trajectories in the configuration space is lost. Therefore, in order to compute the communication energy, one must take into account all the possible transitions in the configuration space. The parallel dynamics studied in the present paper belongs to the family of Probabilistic Cellular Automata (PCA) introduced by [De]. In these lattice models the single site probabilistic updating rule used is local, in the sense that it depends only on the value of the variables in a finite subset of the lattice containing the site itself. A difficulty arising in the study of PCA is the existence of many local minima in which the system can be trapped on its way to the stable phase. The zero temperature dynamics has indeed a large variety of fixed points and cyclic pairs. Examples of cyclic pairs are flip-flop chessboard-like configurations.

The paper [CN] represents the first rigorous study of metastability in a parallel dynamics setup. The model studied in $[\mathrm{CN}]$ is a PCA without self-interaction and can be considered the parallel implementation of the kinetic Ising model. For a comparison between Probabilistic Cellular Automata and serial dynamics in the context of metastability see $[\mathrm{BCLS}],[\mathrm{CN}],[\mathrm{CNS}]$ and $[\mathrm{CNS} 2]$. In [CN] the following three metastability questions have been addressed: 1) what is the transition time between the metastable state $-\underline{1}$ (configuration with all minuses) and the stable state $+\underline{1}$ (configuration with all pluses)? 2) What is the large (critical) fluctuation needed by the system in order to perform the transition towards the equilibrium? 3) Which are the typical paths the system follows during the excursion from the metastable equilibrium $-\underline{1}$ to the stable one $+\underline{1}$ ?

In $[\mathrm{CN}]$ the metastability scenario differs from the one of the kinetic Ising model: starting from -1 the system visits with probability 1 the check-board phase $\mathcal{C}$ before reaching the stable phase $+\underline{1}$. Moreover, the saddles set is richer than expected since the wells of the metastable states $-\underline{1}$ and $\mathcal{C}$ have the same depth.

In the present paper instead, we study a reversible PCA with self-interaction, whose presence makes the single-site updating weights differ from the kinetic Ising's counterpart. The addition of the self-interaction changes again completely the metastability scenario: the check-board $\mathcal{C}$ is not anymore a metastable state. However, in the proof of the the 
transition time the chessboard pairs configuration have to be controlled very carefully. The metastable behavior of this model has been investigated on heuristic and numerical grounds in [BCLS] and rigorously in [CNS]. In [CNS2] the authors introduce a class of PCA depending on a tuning parameter for the strength of the self-interaction in this way one can generate different models with completely different metastability scenarios. The role played by the intermediate metastable state (check-board phase) changes as the selfinteraction is varied. Surprisingly, for some range of the self-interaction parameter results similar to those found for the Blume-Capel model are obtained. Hence, PCA present a non obvious metastable behavior, whose complexity makes this class of models physically very interesting.

The results proven in the present paper can be considered the first attempt to give precise estimates for the mean nucleation time in case of PCA dynamics. In fact, our goal is to improve the already established results in [CNS] by using the potential theoretic approach to metastability instead of large deviation methods (see [BEGK] and [Bo]). Indeed, in [CNS] only the asymptotic behavior of the exponential rates is obtained, while in the present paper we compute the precise prefactors up to multiplicative errors that tend to 1 as the temperature goes to zero. The key ingredient is the reduction of the metastability problem to the computation of capacities, verifying manageable variational principles. In fact, the definition of metastable points can be given in terms of capacities and, once the set of metastable points is identified, precise asymptotics for the mean exit time from each metastable state follows via general theorems. Hence, the basic difficulty is to obtain sharp upper and lower bounds on capacities. However, upper bounds follow from the Dirichlet variational principle, which represents a capacity as an infimum over a class of test functions (see [BEGK]). Furthermore, lower bounds can be derived using another variational principle due to Berman and Konsowa (see $[\mathrm{BK}],[\mathrm{BHS}]$ and $[\mathrm{BBI}]$ ), which represents a capacity as a supremum over a class of unit flows. In this sense, the two variational principles complete each others. The present paper shows the power of potential theoretic approach to metastability: with almost the same model-dependent input of $[\mathrm{CNS}]$ we derive sharp estimates for the nucleation time. Moreover, it also illustrates the application of the powerful Berman Konsowa variational principle to a finite volume parallel dynamics.

The application of the potential approach to our model requires three model dependent results proved in [CNS]: (1) the solution of the global variational problem for all the paths connecting the metastable and the stable state, i.e., the computation of the communication energy; (2) a sort of recurrence property stating that, starting from each configuration different from the metastable and the stable state, it is possible to reach a configuration at lower energy following a path with an energy cost strictly smaller than the communication energy; (3) determination of particular set of configurations, i.e., critical configurations for the transition from metastable to stable state and the neighbor of this set. This set of critical configurations is typically visited by the system during its excursion from the metastable to the stable state. The critical configurations play the role of the saddle configurations in the energy landscape and represent the most important part of the typical tube of trajectories the system follows during the transition. 
Points (1) and (2) are needed to prove that there are only two configurations belonging to the metastable set, defined according to Definition 5.2: -1 , the proper metastable state, and $+\underline{1}$, the stable state. This recurrence property prevents the presence of deep wells where the system can be trapped for long time. Points (1) and (3) are used in the calculation of the capacity between $-\underline{1}$ and $+\underline{1}$. In particular, the prefactor of the nucleation time is related to the cardinality of the saddles set studied in (3).

The outline of the paper is as follows. In Sect. 2 we define the model. In Sect. 3 we state our main theorem on the nucleation time (Theorem 3.1). In Sect. 5 we review the potential theoretic approach to metastability. In particular, we recall two variational principles, namely (5.23) and (5.28), yielding computable upper and lower bounds for the capacities, respectively. Moreover, we state Theorem 5.2 which relates the mean nucleation time to the capacity between the metastable state and the stable state. Sect. 6 is devoted to the proof of Theorem 3.1. Theorem 6.4 guarantees that the dynamics has no deep wells inside where the system can be trapped. Since this result is a corollary of the recurrence properties proven in [CNS], we recall these results in Theorem 6.3. Thus, in Theorem 6.5 sharp estimates for the capacity between $-\underline{1}$ and $+\underline{1}$ are obtained via suitable upper and lower bounds.

\section{Probabilistic Cellular Automata}

In this section we introduce the basic notation, define the class models of reversible Probabilistic Cellular Automata, and the specific one that will be studied in the sequel.

\subsection{The lattice and the configuration space}

First of all for $x=\left(x_{1} ; x_{2}\right) \in \mathbb{R}^{2}$ we set $|x|:=\left|x_{1}\right|+\left|x_{2}\right|$. The spatial structure is modeled by the two-dimensional finite square $\Lambda \subset \mathbb{Z}^{2}$ of side length $L$ with periodic boundary condition namely, by the torus $\Lambda$. We consider $\Lambda$ endowed with the lattice distance $\mathrm{d}(x, y):=|x-y|$.

We say that $x, y \in \Lambda$ are nearest neighbors iff $\mathrm{d}(x, y)=1$. For $X \subset \Lambda$ we let $\partial X:=$ $\left\{x \in X^{\mathrm{c}}: \mathrm{d}(x, X)=1\right\}$ be the external boundary of $X$ and $\bar{X}:=X \cup \partial X$ be the closure of $X$. Two sets $X, Y \subset \Lambda$ are said to be not interacting if and only if $\mathrm{d}(X, Y) \geq 3$.

Let $x \in \Lambda$; for $\ell_{1}, \ell_{2}$ positive integers we let $Q_{\ell_{1}, \ell_{2}}(x):=\left\{y \in \Lambda: x_{1} \leq y_{1} \leq\right.$ $\left.x_{1}+\left(\ell_{1}-1\right), x_{2} \leq y_{2} \leq x_{2}+\left(\ell_{2}-1\right)\right\}$ be the rectangle of side lengths $\ell_{1}$ and $\ell_{2}$ with $x$ the site of the upper-left corner. For $X \subset \Lambda$ and $\ell>0$ we set $B_{\ell}(X):=\{y \in \Lambda: \mathrm{d}(X, y) \leq \ell\}$. If $\ell=1$ we shall write $B(X)$ for $B_{1}(X)$; note that $B(X)=\bar{X}$. If $x \in \Lambda$ we write $B_{\ell}(x)$ for $B_{\ell}(\{x\})$, note that $B_{\ell}(x)$ is the ball of radius $\ell$ centered at $x$. Finally, we remark that $B(x)$ is the five site cross centered at $x \in \Lambda$.

The single spin state space is given by the finite set $\mathcal{S}_{0}:=\{-1,+1\}$ which we consider endowed with the discrete topology; the associated Borel $\sigma$-algebra is denoted by $\mathcal{F}_{0}$. The configuration space in $X \subset \Lambda$ is defined as $\mathcal{S}_{X}:=\mathcal{S}_{0}^{X}$ and considered equipped with the product topology and the corresponding Borel $\sigma$ algebra $\mathcal{F}_{X}$. The model and the related quantities that will be introduced later on will all depend on $\Lambda$, but since $\Lambda$ is fixed it will be dropped from the notation; in this spirit we let $\mathcal{S}_{\Lambda}=: \mathcal{S}$ and $\mathcal{F}_{\Lambda}=: \mathcal{F}$. 
Given $Y \subset X \subset \Lambda$ and $\sigma:=\left\{\sigma_{x} \in \mathcal{S}_{\{x\}}, x \in X\right\} \in \mathcal{S}_{X}$, we denote by $\sigma_{Y}$ the restriction of $\sigma$ to $Y$ namely, $\sigma_{Y}:=\left\{\sigma_{x}, x \in Y\right\}$. Moreover, given $\sigma \in \mathcal{S}$ and $x \in \Lambda$, we denote by $\sigma^{x}$ the configuration such that $\sigma^{x}(x)=-\sigma(x)$ and $\sigma^{x}(y)=\sigma(y)$ for $y \neq x$.

\subsection{The model: Probabilistic Cellular Automaton}

Let $\beta>0$ and $h \in \mathbb{R}$ such that $|h|<1$ and $2 / h$ is not integer, we consider the Markov chain on $\mathcal{S}$ with transition matrix

$$
p(\sigma, \eta):=\prod_{x \in \Lambda} p_{x, \sigma}(\eta(x)) \quad \forall \sigma, \eta \in \mathcal{S}
$$

where, for each $x \in \Lambda$ and $\sigma \in \mathcal{S}, p_{x, \sigma}(\cdot)$ is the probability measure on $\mathcal{S}_{\{x\}}$ defined as follows

$$
p_{x, \sigma}(s):=\frac{1}{1+\exp \left\{-2 \beta s\left(S_{\sigma}(x)+h\right)\right\}}=\frac{1}{2}\left[1+s \tanh \beta\left(S_{\sigma}(x)+h\right)\right]
$$

with $s \in\{-1,+1\}$ and

$$
S_{\sigma}(x):=\sum_{y \in V(x)} \sigma(y)
$$

where $V(x)$ is a suitable neighborhood of the site $x$. Note that for $x$ and $s$ fixed $p_{x, \cdot}(s) \in$ $\mathcal{F}_{V(x)}$ namely, the probability $p_{x, \sigma}(s)$ for the spin at site $x$ to be equal to $s$ depends only on the values of the spins of $\sigma$ inside the the neighborhood $V(x)$ of $x$. The normalization condition $p_{x, \sigma}(s)+p_{x, \sigma}(-s)=1$ is trivially satisfied. We study the metastability behavior of the PCA model, for (2.3) as

$$
V(x):=B(x)
$$

The choice (2.4) corresponds to the model studied in [CNS], note that the selfinteraction of the spin at $x$ is taken into account.

Such a Markov chain on the finite space $\mathcal{S}$ is an example of reversible Probabilistic Cellular Automaton (PCA). Let $n \in \mathbb{N}$ be the discrete time variable and $\sigma_{n} \in \mathcal{S}$ denote the state of the chain at time $n$, the configuration at time $n+1$ is chosen according to the law $p\left(\sigma_{n}, \cdot\right)$, see $(2.1)$, hence all the spins are updated simultaneously and independently at any time. Finally, given $\sigma \in \mathcal{S}$ we consider the chain with initial configuration $\sigma_{0}=$ $\sigma$, we denote with $\mathbb{P}_{\sigma}$ the probability measure on the space of trajectories, by $\mathbb{E}_{\sigma}$ the corresponding expectation value, and by

$$
\tau_{A}^{\sigma}:=\inf \left\{t>0: \sigma_{t} \in A\right\}
$$

the first hitting time on $A \subset \mathcal{S}$; we shall drop the initial configuration from the notation (2.5) whenever it is equal to $-\underline{1}$, we shall write $\tau_{A}$ for $\tau_{A}^{-\underline{1}}$, namely.

\subsection{The stationary measure}

It is easy to prove (see [De]) that the PCA (2.1) is reversible with respect to the finite volume Gibbs measure $\mu(\sigma):=\exp \{-\beta H(\sigma)\} / Z$ with $Z:=\sum_{\eta \in \mathcal{S}} \exp \{-\beta H(\eta)\}$ and

$$
H(\sigma):=H_{\beta, h}(\sigma):=-h \sum_{x \in \Lambda} \sigma(x)-\frac{1}{\beta} \sum_{x \in \Lambda} \log \cosh \left[\beta\left(S_{\sigma}(x)+h\right)\right]
$$


In other words the detailed balance condition

$$
p(\sigma, \eta) e^{-\beta H(\sigma)}=p(\eta, \sigma) e^{-\beta H(\eta)}
$$

is satisfied for any $\sigma, \eta \in \mathcal{S}$, and, as a consequence, the measure $\mu$ is stationary for the PCA (2.1). Since the Hamiltonian has the form (2.6) we shall refer to $1 / \beta$ as to the temperature and to $h$ as to the magnetic field. However, the dependence of the Hamiltonian on $\beta$ makes the definition of ground states not completely trivial. Considering that the ground states are those configurations on which the Gibbs measure $\mu$ is concentrated when $\beta \rightarrow \infty$, they can be defined as the minima of the energy:

$$
E(\sigma):=\lim _{\beta \rightarrow \infty} H(\sigma)=-h \sum_{x \in \Lambda} \sigma(x)-\sum_{x \in \Lambda}\left|S_{\sigma}(x)+h\right|
$$

Let $\mathcal{X} \subset \mathcal{S}$, if the energy $E$ is constant on $\mathcal{X}$ namely, if all the configurations in $\mathcal{X}$ have the same energy, we shall misuse the notation by writing $E(\mathcal{X})$ for $E(\sigma)$ with $\sigma \in \mathcal{X}$.

\subsection{Communication Energy}

In our model the energy difference between two configurations $\sigma$ and $\eta$ is not sufficient to establish if the system prefers to jump from $\sigma$ to $\eta$ or vice versa. Indeed, there are pairs of configurations such that the system sees an energetic barrier in both directions. For this reason we associate a sort of communication height $\mathcal{H}(\sigma, \eta)$ to each pair of configurations $\sigma, \eta \in \mathcal{S}$. More precisely we extend the Hamiltonian (2.6) to $H: \mathcal{S} \cup \mathcal{S} \times \mathcal{S} \rightarrow \mathbb{R}$ so that

$$
\mathcal{H}(\sigma, \eta):=H(\sigma)-\frac{1}{\beta} \log p(\sigma, \eta)
$$

We consider the communication energy

$$
\mathcal{E}(\sigma, \eta):=\lim _{\beta \rightarrow \infty} \mathcal{H}(\sigma, \eta) \geq \max \{E(\sigma), E(\eta)\}
$$

and the transition rate

$$
\Delta(\sigma, \eta):=\mathcal{E}(\sigma, \eta)-E(\sigma)=\sum_{\substack{x \in \Lambda: \\ \eta(x)\left(S_{\sigma}(x)+h\right)<0}} 2\left|S_{\sigma}(x)+h\right| \geq 0
$$

where in the last equality we have used the definition (2.8) of $E(\sigma),(2.9),(2.1)$, and (2.2). We state a simple Lemma that relates the communication height $\mathcal{H}(\sigma, \eta)$ to the communication energy $\mathcal{E}(\sigma, \eta)$.

Lemma 2.1 For any $\sigma, \eta \in \mathcal{S}$ we have:

$$
\mathcal{H}(\sigma, \eta)=\mathcal{E}(\sigma, \eta)+\frac{|\Lambda| \log 2}{\beta}
$$


We will prove the previous lemma in Sect. 4. We give now some useful definitions. A finite sequence of configurations $\omega=\left\{\omega_{1}, \ldots, \omega_{n}\right\}$ is called a path with starting configuration $\omega_{1}$ and ending configuration $\omega_{n}$; we denote the length of a path $|\omega|:=n$. Given a path $\omega$ we define the height along $\omega$ as

$$
\Phi_{\omega}:= \begin{cases}E\left(\omega_{1}\right) & \text { if }|\omega|=1 \\ \max _{i=1, \ldots,|\omega|-1} \mathcal{E}\left(\omega_{i}, \omega_{i+1}\right) & \text { otherwise }\end{cases}
$$

Given two configurations $\sigma, \eta \in \mathcal{S}$, we denote by $\Theta(\sigma, \eta)$ the set of all the paths $\omega$ starting from $\sigma$ and ending in $\eta$. The minimax between $\sigma$ and $\eta$ is defined as

$$
\Phi(\sigma, \eta):=\min _{\omega \in \Theta(\sigma, \eta)} \Phi_{\omega}
$$

\section{Main results: sharp description of nucleation}

We pose now the problem of metastability, we state the related theorem for the exit time for the model in (2.1) with $0<h<1$. Suppose that the system is prepared in the state $\sigma_{0}=-\underline{1}$, where $-\underline{1}$ is the configuration with all the sites with spin -1 , and let the dynamics evolve according with (2.1), we want to study the transition towards the phase $+\underline{1}$, the configuration with all spin +1 . Let us consider the critical length $\lambda$ defined as follows:

$$
\lambda:=\left\lfloor\frac{2}{h}\right\rfloor+1
$$

where, for any positive real $x$, we denote by $\lfloor x\rfloor$ the integer part of $x$, i.e., the largest integer smaller than or equal to $x$. We choose $h$ to be such that $2 / h$ is not integer in order to avoid ties (see [CNS], Sect. 2.3).

In order to state the main theorem for the PCA, we define the following activation energy of the configurations that trigger the nucleation:

$$
\Gamma_{1}:=-4 h \lambda^{2}+4(4+h) \lambda+2-6 h
$$

corresponding to the choice of $V$ in 2.4. In [CNS] it was proven that the random variable $(1 / \beta) \log \tau_{+\underline{1}}$ converges in probability to $\Gamma_{1}$ as $\beta \rightarrow \infty$ and that the logarithm of the mean value of $\tau_{+\underline{1}}$ divided by $\beta$ converges to $\Gamma_{1}$ in the same limit. Moreover, before reaching the stable state $+\underline{1}$, the system started at $-\underline{1}$ must necessarily visit a set of critical droplets $\mathcal{C}$. In the present paper we sharper the results of [CNS], computing the the mean transition time up to sub-exponential prefactors. Indeed, we have:

Theorem 3.1 Consider the probabilistic cellular automaton (2.1), for $h>0$ small enough, we have:

$$
\begin{array}{ll}
\text { i) } & \mathbb{E}_{-\underline{1}}\left(\tau_{+\underline{1}}\right)=\frac{1}{K_{1}} e^{\beta \Gamma_{1}}[1+o(1)] \\
\text { ii) } & \mathbb{P}_{-\underline{1}}\left(\tau_{+\underline{1}}>t \mathbb{E}_{-\underline{1}} \tau_{+\underline{1}}\right)=[1+o(1)] e^{-t[1+o(1)]}, \quad t \geq 0
\end{array}
$$


for $\beta$ large enough and where

$$
K_{1}=2^{-|\Lambda|} 8|\Lambda|(\lambda-1)
$$

\section{Proof of Lemma 2.1}

By using (2.9), (2.1), (2.2), and (2.11) we get:

$$
\begin{aligned}
\mathcal{H}(\sigma, \eta) & -H(\sigma)-[\mathcal{E}(\sigma, \eta)-E(\sigma)] \\
= & \frac{1}{\beta} \sum_{x \in \Lambda} \log \left(1+e^{-2 \beta \eta(x)\left[S_{\sigma}(x)+h\right]}\right)+\sum_{\substack{x \in \Lambda: \\
\eta(x)\left(S_{\sigma}(x)+h\right)<0}} 2 \eta(x)\left[S_{\sigma}(x)+h\right] \\
= & \frac{1}{\beta} \sum_{\substack{x \in \Lambda: \\
\eta(x)\left(S_{\sigma}(x)+h\right)>0}} \log \left(1+e^{-2 \beta \eta(x)\left[S_{\sigma}(x)+h\right]}\right)+\frac{1}{\beta} \sum_{\substack{x \in \Lambda: \\
\eta(x)\left(S_{\sigma}(x)+h\right)<0}} \log \left(1+e^{-2 \beta \eta(x)\left[S_{\sigma}(x)+h\right]}\right) \\
& +\sum_{\substack{x \in \Lambda: \\
\eta(x)\left(S_{\sigma}(x)+h\right)<0}} 2 \eta(x)\left[S_{\sigma}(x)+h\right] \\
= & \frac{1}{\beta} \sum_{\substack{x \in \Lambda: \\
\eta(x)\left(S_{\sigma}(x)+h\right)>0}} \log \left(1+e^{-2 \beta \eta(x)\left[S_{\sigma}(x)+h\right]}\right)+\frac{1}{\beta} \sum_{\substack{x \in \Lambda: \\
\eta(x)\left(S_{\sigma}(x)+h\right)<0}} \log \left(e^{+2 \beta \eta(x)\left[S_{\sigma}(x)+h\right]}+1\right) \\
= & \frac{1}{\beta} \sum_{x \in \Lambda} \log \left(1+e^{-2 \beta\left|S_{\sigma}(x)+h\right|}\right)
\end{aligned}
$$

Moreover, since

$$
\begin{aligned}
H(\sigma) & :=-\frac{1}{\beta} \sum_{x \in \Lambda} \log \left(\cosh \left(\beta\left(S_{\sigma}(x)+h\right)\right)\right)-h \sum_{x} \sigma(x) \\
& =-\frac{1}{\beta} \sum_{x \in \Lambda} \log \left(\cosh \left(\beta\left|S_{\sigma}(x)+h\right|\right)\right)-h \sum_{x} \sigma(x) \\
E(\sigma) & :=-h \sum_{x} \sigma(x)-\sum_{x}\left|S_{\sigma}(x)+h\right|
\end{aligned}
$$

we have for the energy:

$$
\begin{aligned}
H(\sigma)-E(\sigma) & =-\frac{1}{\beta}\left[\sum_{x \in \Lambda} \log \left(\cosh \left(\beta\left|S_{\sigma}(x)+h\right|\right)\right)-\beta \sum_{x}\left|S_{\sigma}(x)+h\right|\right] \\
& =-\frac{1}{\beta}\left[\sum_{x}\left(\log \left(\cosh \left(\beta\left|S_{\sigma}(x)+h\right|\right)\right)-\log \exp \left(\beta\left|S_{\sigma}(x)+h\right|\right)\right)\right] \\
& =-\frac{1}{\beta}\left[\sum_{x}\left(\log \frac{e^{\beta\left|S_{\sigma}(x)+h\right|}+e^{-\beta\left|S_{\sigma}(x)+h\right|}}{2 e^{\beta\left|S_{\sigma}(x)+h\right|}}\right)\right] \\
& =-\frac{1}{\beta}\left[\sum_{x}\left(\log \frac{1+e^{-2 \beta\left|S_{\sigma}(x)+h\right|}}{2}\right)\right]
\end{aligned}
$$


By summing (4.20) and (4.21) we get (2.12).

\section{Review on Potential Theoretic approach}

The proof of the Theorem (3.1) is based on the potential-theoretic approach to metastability developed in [BEGK]. In this approach a key role is played by the notion of capacity between two sets of configurations, which we are going to recall.

\subsection{Capacity}

For any $h: \mathcal{S} \rightarrow \mathbb{R}$, we define the Dirichlet form in the following way:

$$
\mathfrak{E}(h)=\frac{1}{2} \sum_{\sigma, \eta \in \mathcal{S}} \mu(\sigma) p(\sigma, \eta)[h(\sigma)-h(\eta)]^{2}=\frac{1}{2} \sum_{\sigma, \eta \in \mathcal{S}} \frac{1}{Z} e^{-\beta \mathcal{H}(\sigma, \eta)}[h(\sigma)-h(\eta)]^{2}
$$

where $Z$ is the partition function defined in Sect. 2.3. From the definition (2.9) of communication energy, we have

$$
\mu(\sigma) p(\sigma, \eta)=\mu(\sigma) e^{-\beta(\mathcal{H}(\sigma, \eta)-H(\sigma))}=\frac{1}{Z} e^{-\beta \mathcal{H}(\sigma, \eta)}
$$

Given two non-empty disjoint sets $\mathcal{A}$ and $\mathcal{B}$, the capacity of the pair $\mathcal{A}, \mathcal{B}$ is defined as follows:

$$
\operatorname{CAP}(\mathcal{A}, \mathcal{B}):=\min _{\substack{h:\left.\mathcal{S} \rightarrow[0,1] \\ h\right|_{\mathcal{A}}=1,\left.h\right|_{\mathcal{B}}=0}} \mathfrak{E}(h)
$$

By (5.23) follows that the capacity is a symmetric function of the sets $\mathcal{A}$ and $\mathcal{B}$. The right hand side of (5.23) has a unique minimizer $h_{\mathcal{A}, \mathcal{B}}^{*}$ called equilibrium potential of the pair $\mathcal{A}, \mathcal{B}$ given by

$$
h_{\mathcal{A}, \mathcal{B}}^{*}(\eta)=\mathbb{P}_{\eta}\left(\tau_{\mathcal{A}}<\tau_{\mathcal{B}}\right)
$$

for any $\eta \notin \mathcal{A} \cup \mathcal{B}$. Hence, inserting a general test function $h$ in the Dirichlet form, one obtains an upper bound for the capacity. Obviously, the closer $h$ is to the equilibrium potential, the sharper is the bound.

A remarkable property of capacity is that it can be characterized also by another variational principle, useful for giving a lower bound. It is given in term of unitary flows. For the sake of completeness, we recall the main definitions and results given in [BHS] and [BBI] (pg. 1550-1551). In the following, it will be convenient to think of $\mathcal{S}$ as the vertex set of a graph $(\mathcal{S}, \mathcal{L})$ whose edge set $\mathcal{L}$ consists of all pairs $(\sigma, \eta), \sigma, \eta \in \mathcal{S}$, for which $p(\sigma, \eta)>0$.

Definition 5.1 Given two non-empty disjoint sets $\mathcal{A}, \mathcal{B} \subset \mathcal{S}$, a loop-free non-negative unit flow, $f$, from $\mathcal{A}$ to $\mathcal{B}$ is a function $f: \mathcal{L} \rightarrow[0, \infty)$ such that: 
(a) $(f(e)>0 \Longrightarrow f(-e)=0) \forall e \in \mathcal{E}$.

(b) f satisfies Kirchoff's law:

$$
\sum_{\sigma^{\prime} \in \mathcal{S}} f\left(\sigma, \sigma^{\prime}\right)=\sum_{\sigma^{\prime \prime} \in \mathcal{S}} f\left(\sigma^{\prime \prime}, \sigma\right), \quad \forall \sigma \in \mathcal{S} \backslash(\mathcal{A} \cup \mathcal{B})
$$

(c) $f$ is normalized:

$$
\sum_{\sigma \in \mathcal{A}} \sum_{\sigma^{\prime} \in \mathcal{S}} f\left(\sigma, \sigma^{\prime}\right)=1=\sum_{\sigma \in \mathcal{S}} \sum_{\sigma \in \mathcal{B}} f\left(\sigma^{\prime \prime}, \sigma\right) .
$$

(d) Any path from $\mathcal{A}$ to $\mathcal{B}$ along edges e such that $f(e)>0$ is self-avoiding.

The space of all loop-free non-negative unit flows from $\mathcal{A}$ to $\mathcal{B}$ is denoted by $\mathbb{U}_{\mathcal{A}, \mathcal{B}}$.

A loop-free non-negative unit flow $f$ is naturally associated with a probability measure $\mathbb{P}^{f}$ on self-avoiding paths, $\gamma$. To see this, define $F(\sigma)=\sum_{\sigma^{\prime} \in \mathcal{S}} f\left(\sigma, \sigma^{\prime}\right), \sigma \in \mathcal{S} \backslash \mathcal{B}$. Then $\mathbb{P}^{f}$ is the Markov chain $\left(\sigma_{n}\right)_{n \in \mathbb{N}_{0}}$ with initial distribution $\mathbb{P}^{f}\left(\sigma_{0}\right)=F\left(\sigma_{0}\right) 1_{\mathcal{A}}\left(\sigma_{0}\right)$, transition probabilities

$$
q^{f}\left(\sigma, \sigma^{\prime}\right)=\frac{f\left(\sigma, \sigma^{\prime}\right)}{F(\sigma)}, \quad \sigma \in \mathcal{S} \backslash \mathcal{B},
$$

such that the chain is stopped upon arrival in $\mathcal{B}$. In terms of this probability measure, we have the following proposition:

Proposition 5.1 Let $\mathcal{A}, \mathcal{B} \subset \mathcal{S}$ be two non-empty disjoint sets. Then, with the notation introduced above,

$$
\operatorname{CAP}(\mathcal{A}, \mathcal{B})=\sup _{f \in \mathbb{U}_{\mathcal{A}, \mathcal{B}}} \mathbb{E}^{f}\left(\left[\sum_{e \in \gamma} \frac{f\left(e_{l}, e_{r}\right)}{\mu\left(e_{l}\right) p\left(e_{l}, e_{r}\right)}\right]^{-1}\right)
$$

where $e=\left(e_{l}, e_{r}\right)$ and the expectation is with respect to $\gamma$.

The nice feature of this variational principle is that any flow gives a computable lower bound. In this sense (5.23) and (5.28) complement each other.

Remark. As showed in [BNS], the lower bounds for the capacity could have been obtained using the monotonicity of capacities in the transition probabilities (Raighleys principle). However, several recent papers (see $[\mathrm{BBI}],[\mathrm{BHS}]$ ) showed the power of Berman-Konsowa variational principle in models where entropy is an issue. Despite in our case entropy does not play any role, our finite-volume setting offers the possibility to illustrate the use of this variational principle in a simpler framework, providing an intuitive an elegant way of obtaining the precise lower bound.

\subsection{Relation between capacity and the mean hitting time}

We want now to recall a theorem from [BEGK] that links mean hitting times and capacities. Indeed, we define the set of metastable configuration $\mathcal{M}$ : 
Definition 5.2 Consider a family of Markov chains, indexed by $\beta$, on a finite state space $\mathcal{S}$. A set $\mathcal{M} \subseteq \mathcal{S}$ is called metastable if

$$
\lim _{\beta \rightarrow \infty} \frac{\max _{\eta \notin \mathcal{M}} \mu(\eta)[\operatorname{CAP}(\eta, \mathcal{M})]^{-1}}{\min _{\eta \in \mathcal{M}} \mu(\eta)[\operatorname{CAP}(\eta, \mathcal{M} \backslash \eta)]^{-1}}=0
$$

Definition 5.3 The valley $A(\sigma)$ for a configuration $\sigma \in \mathcal{M}$ is defined as

$$
A(\sigma):=\left\{\zeta \in \mathcal{S}: \mathbb{P}_{\zeta}\left(\tau_{\sigma}=\tau_{\mathcal{M}}\right)=\sup _{\eta \in \mathcal{M}} \mathbb{P}_{\zeta}\left(\tau_{\eta}=\tau_{\mathcal{M}}\right)\right\}
$$

With these definitions, we are able to write the mean hitting times in terms of the capacities, that satisfies a manageable variational principle. In fact, the following theorem holds:

Theorem 5.2 Let $\sigma \in \mathcal{M}$ and $J \subseteq \mathcal{M} \backslash x$ be such that for all $m \in \mathcal{M} \backslash(J \cup x)$ either $\mu(m) \ll \mu(\sigma)$ or $\operatorname{CAP}(m, J) \gg \operatorname{CAP}(m, \sigma)$. Then

$$
\begin{aligned}
\text { i) } & \mathbb{E}_{\sigma} \tau_{J}=\frac{\mu(A(\sigma))}{\operatorname{CAP}(\sigma, J)}(1+o(1)) \\
\text { ii) } & \mathbb{P}_{\sigma}\left(\tau_{J}>t \mathbb{E}_{\sigma} \tau_{J}\right)=[1+o(1)] e^{-t[1+o(1)]} t \geq 0
\end{aligned}
$$

for $\beta$ large enough.

Hence, the strategy for proving the main theorem will be to identify the metastable sets (i.e., $-\underline{1},+\underline{1}$ ), and to give sharp estimates for the capacities among the elements of this set, via a suitable application of the variational principle for the Dirichlet form.

\section{Proof of Theorem 3.1}

In order to prove the theorem, we first show that the metastable set $\mathcal{M}$ is $\{-\underline{1},+\underline{1}\}$, and that $\mu(A(-\underline{1}))=\mu(-\underline{1})(1+o(1))$ for $\beta$ large enough. Hence, we give sharp estimates on $\operatorname{CAP}(-\underline{1},+\underline{1})$ and we conclude the proof by using Theorem 5.2.

6.1. Metastable set $\mathcal{M}=\{-\underline{1},+\underline{1}\}$

Let us give the following definitions:

Definition 6.4 (Saddles) 1. We denote by $\mathcal{P}$ the set of the protocritical droplets containing the configurations with all the spins equal to -1 excepted those in a rectangle of sides $\lambda-1$ and $\lambda$ in a neighboring site adjacent to one of the longest sides. 


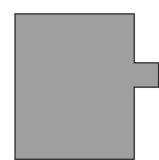

$\eta \in \mathcal{P}$

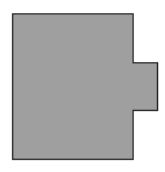

$\zeta \in \mathcal{C}$

Figure 1: Protocritical and critical droplets

2. we denote with $\mathcal{C}$ the set of the critical droplets consisting in the configurations with all the spins equal to -1 excepted those in a rectangle of sides $\lambda-1$ and $\lambda$ in a pair of neighboring sites adjacent to one of the longest sides.

We restate a theorem from [CNS], giving a recurrence property on the set $\{-\underline{1},+\underline{1}\}$, and estimate in probability of the first hitting time in the stable state.

Theorem 6.3 [CNS] Recall $\Gamma_{1}$ has been defined in (3.16) and suppose $h>0$ is chosen small enough; then

1. for any $\sigma \in \mathcal{S} \backslash\{-\underline{1}\}$ we have

$$
\Phi(\sigma,+\underline{1})-E(\sigma)<\Gamma_{1}
$$

2. we have

$$
\Phi(-\underline{1},+\underline{1})-E(-\underline{1})=\Gamma_{1}
$$

3. for each path $\omega=\left\{\omega_{1}, \ldots, \omega_{n}\right\} \in \Theta(-\underline{1},+\underline{1})$ such that $\Phi_{\omega}=\Gamma_{1}$, then $\mathcal{E}\left(\omega_{i-1}, \omega_{i}\right)=$ $\Gamma_{1}$ iff $\omega_{i-1} \in \mathcal{P}, \omega_{i} \in \mathcal{C}$ and $\omega_{i}=\omega_{i-1}^{x}$ for a suitable $x \in \Lambda$.

Moreover recall the following estimate given in $[\mathrm{BHN}]$ :

Lemma 6.2 [BHN] For every non-empty disjoint sets $\mathcal{A}, \mathcal{B} \subset \mathcal{S}$, there exist constants $0<C_{1} \leq C_{2}<\infty$ (depending on $\left.\mathcal{A}, \mathcal{B}\right)$ such that for all $\beta$ :

$$
C_{1} \leq e^{\beta \Phi(\mathcal{A}, \mathcal{B})} Z \operatorname{CAP}(\mathcal{A}, \mathcal{B}) \leq C_{2}
$$

Recall Definition 5.3 of metastable set $\mathcal{M}$, the following holds

\section{Theorem 6.4}

$$
\mathcal{M}=\{-\underline{1},+\underline{1}\}
$$

\section{Proof}

For simplicity in the sequel we will denote by $C_{i}$ any positive constant non depending on $\beta$. From (6.33) and (6.35), it follows that, for any $\eta \notin\{-\underline{1},+\underline{1}\}$

$$
\mu(\eta)[\operatorname{CAP}(\eta, \mathcal{M})]^{-1} \leq \frac{Z}{C_{1}} \mu(\eta) e^{\beta \phi(\eta,+1)} \leq \frac{e^{-H(\sigma)}}{C_{1}} e^{\beta \phi(\eta, \mathcal{M})} \leq C_{3} e^{\beta\left(\Gamma_{1}-\delta\right)}
$$


for some $\delta>0$, where in the last inequality we used (4.21).

If $\eta \in\{-\underline{1},+\underline{1}\}$, we have

$$
\mu(\eta)[\mathrm{CAP}(-\underline{1},+\underline{1})]^{-1} \geq C_{4} e^{\beta \Gamma_{1}}
$$

Indeed, if $\eta=-\underline{1}$ equation (6.38) follows from (6.35), (6.34) and (4.21), while if $\eta=+\underline{1}$ we can write:

$$
\frac{\mu(+\underline{1})}{\operatorname{CAP}(-\underline{1},+\underline{1})} \geq Z \frac{\mu(+\underline{1})}{C_{2} e^{-\beta \phi(-\underline{1},+\underline{1})}}=\frac{e^{\beta \phi(-\underline{1},+\underline{1})-H(+\underline{1})}}{C_{2}}>C_{5} e^{\beta \Gamma_{1}}
$$

Hence, from (6.38) and (6.37), we get

$$
\frac{\max _{\eta \notin \mathcal{M}} \mu(\eta)[\operatorname{CAP}(\eta, \mathcal{M})]^{-\underline{1}}}{\min _{\eta \in \mathcal{M}} \mu(\eta)[\operatorname{CAP}(\eta, \mathcal{M} \backslash \eta)]^{-1}} \leq C_{6} e^{-\beta \delta}
$$

that concludes the proof. $\square$

We state and prove now a simple lemma regarding the measure of the valley $A(-1)$ of the metastable configuration -1 .

\section{Lemma 6.3}

$$
\mu(A(-\underline{1}))=\mu(-\underline{1})(1+o(1))
$$

for $\beta$ large enough.

\section{Proof}

The valley (5.30) for the metastable configuration $-\underline{1}$ can be written also in the form:

$$
A(-\underline{1}):=\left\{\zeta \in \mathcal{S}: \mathbb{P}_{\zeta}\left(\tau_{-\underline{1}} \leq \tau_{+\underline{1}}\right) \geq \mathbb{P}_{\zeta}\left(\tau_{+\underline{1}} \leq \tau_{-\underline{1}}\right)\right\}
$$

From $[\mathrm{BHN}]$ (see the proof of Proposition 3.2.3), using elementary properties of the equilibrium potential, we have the inclusions:

$$
A_{1}(-\underline{1}) \subseteq A(-\underline{1}) \subseteq A_{2}(-\underline{1})
$$

for $\beta$ large enough and where

$$
\begin{aligned}
& A_{1}(-\underline{1}):=\{\zeta \in \mathcal{S}: \Phi(\zeta,-\underline{1})<\Phi(\zeta,+\underline{1})\} \\
& A_{2}(-\underline{1}):=\{\zeta \in \mathcal{S}: \Phi(\zeta,-\underline{1}) \leq \Phi(\zeta,+\underline{1})\}
\end{aligned}
$$

If $\eta \in A_{2}(-\underline{1})$, by absurdity, is such that $E(\eta) \leq E(-\underline{1})$, then we show that $\Phi(-\underline{1},+\underline{1})<$ $\Gamma_{1}+E(-\underline{1})$, in contradiction with (6.34). In fact, let us consider the paths $\omega_{1}:-\underline{1} \rightarrow \eta$ attaining the minimax $\Phi(-\underline{1}, \eta)$ and $\omega_{2}: \eta \rightarrow+\underline{1}$ attaining the minimax $\Phi(\eta,+\underline{1})$. Merging the path $\omega_{1}$ with $\omega_{2}$ we obtain the path $\omega:-1 \rightarrow+1$. Hence, $\Phi_{\omega}=\max (\Phi(-\underline{1}, \eta), \Phi(\eta,+\underline{1}))=$ $\Phi(\eta,+\underline{1})$, for the definition of the set $A_{2}(-\underline{1})$. By the recurrence property 6.33 :

$$
\Phi(\eta,+\underline{1})<\Gamma_{1}+E(\eta)
$$


so that

$$
\Phi_{\omega}<\Gamma_{1}+E(-\underline{1})=\Phi(-\underline{1},+\underline{1})
$$

that is an absurd from (6.34). In as similar way one can proceed for the set $A_{1}(-\underline{1})$. Hence, for any $\eta \in A(-\underline{1}) \backslash-\underline{1}$, we have $E(\eta)>E(-\underline{1})$ that concludes the proof.

\subsection{Sharp estimates for $\operatorname{CAP}(-\underline{1},+\underline{1})$}

In the case of parallel dynamics the lacking of continuity increases the difficulty of the computation of the communication energy between the metastable and the stable state. Hence in $[\mathrm{CNS}]$ a set $\mathcal{G} \subset \mathcal{S}$ containing $-\underline{1}$, but not $+\underline{1}$ has been constructed in such a way that the evaluation of the transition energy for all the possible transitions from the interior to the exterior of such a set $\mathcal{G}$ was under control. The set $\mathcal{G}$ defined in [CNS] in Sect. 3.2 is constructed using the two mappings $A, B: \mathcal{S} \rightarrow \mathcal{S}$. We recall their definitions for the sake of completeness. The map $A$ flips the first, in lexicographic order, unstable plus spin of $\sigma$, providing that this change corresponds to a decrease in the energy. More explicitly, the map changes the value in $x \in \Lambda$ if $\sigma(x)=+1, \sum_{y \in V(x)} \sigma(y) \leq-1$ and providing that $H\left(\sigma^{x}\right) \leq H(\sigma)$. The effect of the map $A$ is that the number of unstable pluses decrease. We iteratively apply the map $A$ to $\sigma$, until we reach a fixed point, denoted by $\bar{A} \sigma$. For any $\sigma \in \mathcal{S}$ the configuration $B \sigma$ is the single step bootstrap percolation, i.e., it changes the value in $x \in \Lambda$ if the $\sigma(x)=-1$ and $\sum_{y \in V(x)} \sigma(y) \geq-1$.

The iteration of the map $B$ on $\bar{A} \sigma$ converges to a fixed point $\bar{B} \bar{A} \sigma$ in a finite time. The composed mapping is not necessarily the result of the zero temperature dynamics, but has the remarkable property of having the support consisting in well separated rectangles or stripes winding around the torus. We can now define the set $\mathcal{G}$. Denoting by $\cup_{i}^{n(\sigma)} Q_{\ell_{i, 1}, \ell_{i, 2}}\left(x_{i}\right)$ the collection of $n(\sigma)$ pairwise not interacting rectangles (or stripes) being the support of the fixed point $\bar{B} \bar{A} \sigma$ of the composed mapping, we define $\mathcal{G}$ as the set of all configurations $\sigma$ such that the fixed point $\bar{B} \bar{A} \sigma$ is either -1 or $\min \left\{\ell_{i, 1}, \ell_{i, 2}\right\} \leq \lambda-1$ and $\max \left\{\ell_{i, 1}, \ell_{i, 2}\right\} \leq L-2$ for any $i=1, \ldots, n(\sigma)$. Note that the set $\mathcal{G}$ contains the $s u b$ critical configurations (namely, starting from such a configuration the system visits $-\underline{1}$ before $+\underline{1}$ with probability tending to one in the limit $\beta \rightarrow \infty)$ and that configurations $\sigma$ such that $\bar{B} \bar{A} \sigma$ contains plus stripes winding around the torus $\Lambda$ do not belong to $\mathcal{G}$. Following [CNS] we can evaluate the minmax between $\mathcal{G}$ and $\mathcal{G}^{c}$.

\section{Proposition 6.2 [CNS]}

With the definitions above, for $h>0$ small enough and $L=L(h)$ large enough, we have

1. $-\underline{1} \in \mathcal{G},+\underline{1} \in \mathcal{S} \backslash \mathcal{G}$, and $\mathcal{C} \subset \mathcal{S} \backslash \mathcal{G}$;

2. for each $\eta \in \mathcal{G}$ and $\zeta \in \mathcal{S} \backslash \mathcal{G}$ we have $\mathcal{E}(\eta, \zeta) \geq E(-1)+\Gamma_{1}$;

3. for each $\eta \in \mathcal{G}$ and $\zeta \in \mathcal{S} \backslash \mathcal{G}$ we have $\mathcal{E}(\eta, \zeta)=E(-1)+\Gamma_{1}$ if and only if $\zeta \in \mathcal{C}$, $\eta \in \mathcal{P}$ and $\zeta=\eta^{x}$ for a suitable $x \in \Lambda$.

Now we have all the ingredients to prove the sharp estimates for the capacity between $-\underline{1}$ and $+\underline{1}$. 
Theorem 6.5 With the notation of Theorem 3.1, we have:

$$
\operatorname{CAP}(-\underline{1},+\underline{1})=K_{1} \mu(-\underline{1}) e^{-\beta \Gamma_{1}}(1+o(1))
$$

in the limit $\beta \rightarrow \infty$

\section{Proof.}

In order to prove the theorem we give upper and lower bounds to the capacity $\mathrm{CAP}(-\underline{1},+\underline{1})$.

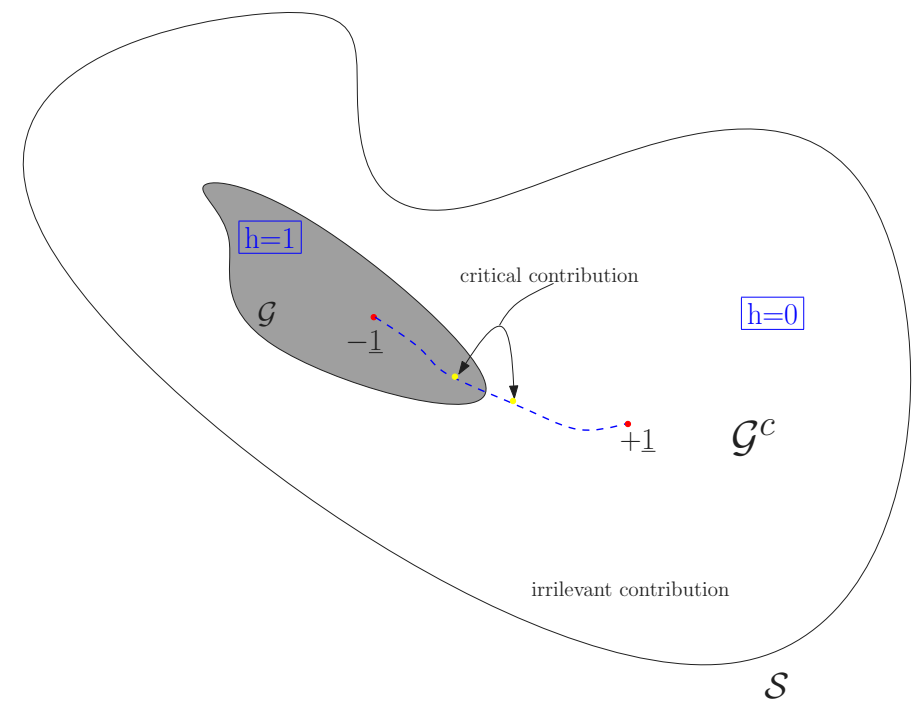

Figure 2: Upper bound

i) Upper bound

We use a general strategy to prove a upper bound by guessing some a-priori properties of the minimizer $h^{\star}$, solving of the variational problem (5.23). We give an upper bound $h^{u}$ to the equilibrium potential $h^{\star}$ choosing a test function in the following way:

$$
h^{u}(\sigma):=\left\{\begin{array}{cc}
1 & \sigma \in \mathcal{G} \\
0 & \sigma \in \mathcal{G}^{c}
\end{array}\right.
$$

Thus

$$
\mathfrak{E}\left(h^{u}\right)=\frac{1}{Z} \sum_{\substack{\sigma \in \mathcal{G}, \eta \in \mathcal{G}^{c}}} e^{-H(\sigma, \eta)}=\frac{1}{Z 2^{|\Lambda|}} \sum_{\substack{\sigma \in \mathcal{G}, \eta \in \mathcal{G}^{c}}} e^{-\beta \mathcal{E}(\sigma, \eta)} \leq \frac{\mu(-\underline{1})}{2^{|\Lambda|}} \sum_{\substack{\sigma \in \mathcal{G}, \eta \in \mathcal{G}^{c}}} e^{-\beta(\mathcal{E}(\sigma, \eta)-E(-1))}
$$

where in the second step we used (2.12) and in the last (4.21). We denote with $N_{1}$ the cardinality of the set of the saddles where the minmax $\Gamma_{1}$ is attained. The l.h.s of (6.44), is bounded as follows: 


$$
\frac{2^{|\Lambda|} \mathfrak{E}\left(h^{u}\right)}{\mu(-\underline{1})} \leq N_{1} e^{-\beta \Gamma_{1}}+|\mathcal{S}| e^{-\beta\left(\Gamma_{1}+\delta\right)}
$$

where $\delta>0$. Indeed $\Phi\left(\mathcal{G}, \mathcal{G}^{c}\right)=\Gamma+E(-\underline{1})$ and, by Proposition 6.2, the minmax is attained only in the transitions between configurations $\eta \in \mathcal{P} \subset \mathcal{G}$ and $\zeta \in \mathcal{C} \subset \mathcal{G}^{c}$. Therefore, for all the other transitions we have $\mathcal{E}(\eta, \zeta)>\Gamma+E(-\underline{1})$.

We denoted with $N_{1}$, the number of the possible ways to choose a protocritical droplet in the lattice with periodic boundary conditions. We know that the set $\mathcal{P}$ of such configurations contains all the rectangles $Q_{\lambda-1, \lambda}(x)$ with a single protuberance adjacent to one of the largest sides. Because of the translational invariance on the lattice, we can associate at each site $x$ two rectangular droplets $Q_{\lambda-1, \lambda}(x)$ and $Q_{\lambda, \lambda-1}(x)$ such that their lower-left corner is in $x$. Considering the periodic boundary conditions, the number of such rectangles is

$$
N_{Q}=2|\Lambda|
$$

For every rectangle the possible transitions between a protocritical droplet and a critical droplet are:

$$
N_{S}=2(2(\lambda-2)+2)
$$

since there are two larger sides and given a single protuberance there are two ways to form a double protuberance if the spin is not in a corner and just one otherwise. Thus

$$
N_{1}=N_{Q} N_{S}=8|\Lambda|(\lambda-1)
$$

Hence,

$$
\operatorname{CAP}(-\underline{1},+\underline{1}) \leq K_{1} \mu(-\underline{1}) e^{-\beta \Gamma}(1+o(1))
$$

for $\beta$ large enough.

ii) Lower bound

In [CNS] (see Section 3.1) we have detailed information about the optimal paths attaining the minmax $\Phi(-\underline{1},+\underline{1})$ for the nucleation of $+\underline{1}$ starting from $-\underline{1}$. We exploit Proposition 5.1 by making a judicious choice for the flow $f$. We distribute indeed the mass of the test flow equally among a suitable subset of optimal paths. More precisely, starting from -1 , we flip with uniform probability in the lattice the spin in the site $x$, obtaining the configuration $\sigma:=-\underline{1}^{(x)}$. From $\sigma$, either we follow with probability $1 / 2$ a deterministic path, along which we nucleate a horizontal quasi-square droplet with support equal to $Q_{\lambda-1, \lambda}(x)$ in the canonical order described in Fig. 3, or with probability $1 / 2$ we nucleate deterministically a vertical quasi-square droplet with support equal to $Q_{\lambda, \lambda-1}(x)$ following Fig. 4 . Once the quasi-square is nucleated, with probability $1 / \lambda$ we flip a site along one of the two longest sides, creating a protuberance. In case the protuberance is on a corner as 
in Fig 5, we continue deterministically to nucleate the square $Q_{\lambda, \lambda}(x)$ enlarging the protuberance by flipping adjacent spins. Otherwise, with probability $1 / 2$ we choose one of the two sites next to the protuberance, creating a double protuberance as described in Fig 6. Hence, we continue to enlarge the protuberance, in the same direction used to change the previous spin, flipping spins adjacent to the chosen site until we reach the corner (see Fig 6). Afterwards, we proceed in similar way flipping adjacent spins until we reach the other corner and we nucleate the droplet with support $Q_{\lambda, \lambda}(x)$. Once we have nucleated the square droplet of side $\lambda$, the two flows for the horizontal and the vertical protocritical droplets merge. Hence, we simply continue invading all the lattice following deterministically a canonical path as described in Fig 7 . We set

$$
K=\lambda(\lambda-1)
$$

and

$$
\nu_{0}=\frac{1}{|\Lambda|}
$$

Moreover, we denote with $\mathcal{P}_{c}$ the set of all the configurations with a protocritical droplet with a protuberance in a corner of one of the longest sides (see the first configuration in Fig 5), and with $\mathcal{P}_{n c}$ the set of all the configurations with a protocritical droplet with a protuberance not in the corner (see the first configuration in Fig. 6). In addition, following the notation introduced in Fig. 3 and in Fig. 4, we denote with $\gamma_{x}^{i}$ a canonical path starting from the site $x$ and nucleating a horizontal protocritical droplet if $i=1$ or a vertical if $i=2$.

Thus, we can define the unitary flow from $-\underline{1}$ to $+\underline{1}$, corresponding to the algorithm 
so far explained:

$$
f\left(\sigma^{\prime}, \sigma^{\prime \prime}\right)= \begin{cases}\nu_{0} & \text { if } \sigma^{\prime}=-\underline{1}, \sigma^{\prime \prime}=-\underline{1}^{(x)}, \text { for some } x \in \Lambda, \\ \frac{\nu_{0}}{2} \quad \text { if } \sigma^{\prime}=\gamma_{x}^{i}(k), \sigma^{\prime \prime}=\gamma_{x}^{i}(k+1), & \text { for some }, x \in \Lambda, i \in\{1,2\}, 1 \leq k \leq K \\ \frac{\nu_{0}}{4 \lambda} \quad \text { if } \sigma^{\prime}=\gamma_{x}^{i}(K), \sigma^{\prime \prime}=\gamma_{x}^{i}(K+1) & \text { for some }, x \in \Lambda, i \in\{1,2\} \\ \frac{\nu_{0}}{4 \lambda} \quad \text { if } \sigma^{\prime}=\gamma_{x}^{i}(K+1), \sigma^{\prime \prime}=\gamma_{x}^{i}(K+2), & \gamma_{x}^{i}(K+1) \in \mathcal{P}_{c}, \text { for some }, x \in \Lambda, i \in\{1,2\} \\ \frac{\nu_{0}}{8 \lambda} \quad \text { if } \sigma^{\prime}=\gamma_{x}^{i}(K+1), \sigma^{\prime \prime}=\gamma_{x}^{i}(K+2), & \gamma_{x}^{i}(K+1) \in \mathcal{P}_{n c}, \text { for some }, x \in \Lambda, i \in\{1,2\} \\ \frac{\nu_{0}}{4 \lambda} \quad \text { if } \sigma^{\prime}=\gamma_{x}^{i}(k), \sigma^{\prime \prime}=\gamma_{x}^{i}(k+1) \\ \quad \gamma_{x}^{i}(K+1) \in \mathcal{P}_{c}, \text { for some }, x \in \Lambda, i \in\{1,2\}, \\ \frac{\nu_{0}}{8 \lambda} \quad \text { if } \sigma^{\prime}=\gamma_{x}^{i}(k), \sigma^{\prime \prime}=\gamma_{x}^{i}(k+1) \\ \quad \gamma_{x}^{i}(K+1) \in \mathcal{P}_{n c}, \text { for some }, x \in \Lambda, i \in\{1,2\}, \\ & K+2 \leq k \leq \lambda^{2}-1 \\ \nu_{0} & \text { if } \sigma^{\prime}=\gamma_{x}^{i}(k), \sigma^{\prime \prime}=\gamma_{x}^{i}(k+1) \\ & \text { for some } x \in \Lambda, i \in\{1,2\}, \lambda^{2} \leq k \leq|\Lambda|-1 \\ 0, \quad & \text { otherwise. }\end{cases}
$$

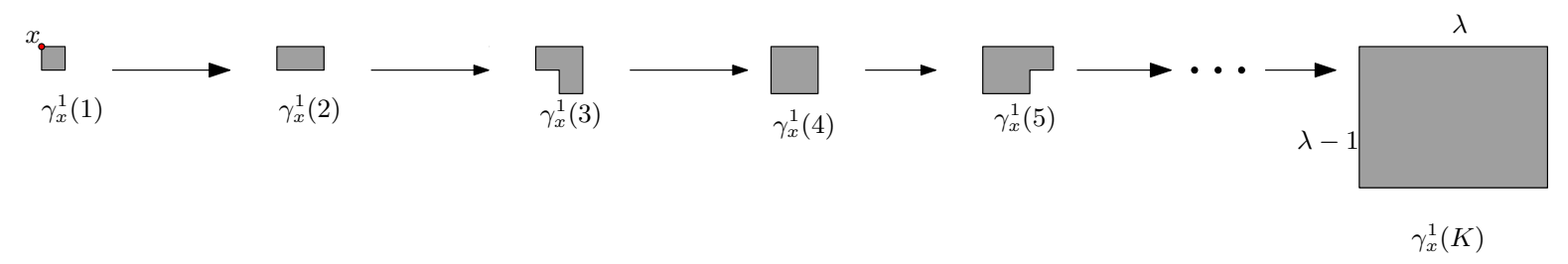

Figure 3: Canonical order to nucleate $\gamma_{x}^{1}(K)$

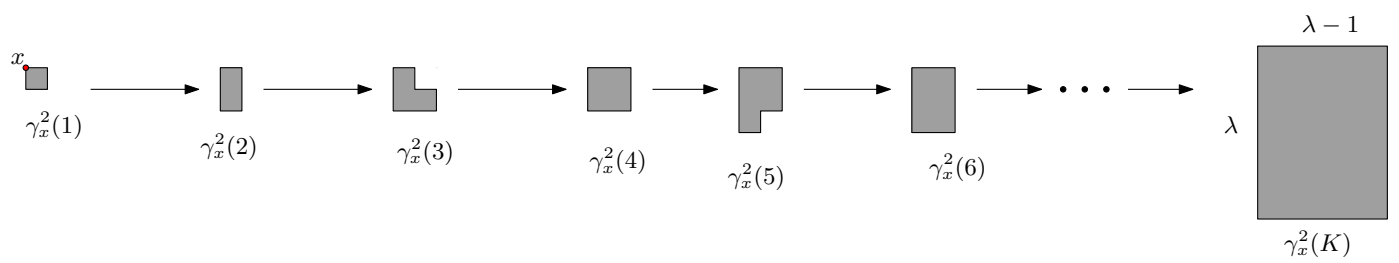

Figure 4: Canonical order to nucleate $\gamma_{x}^{2}(K)$ 


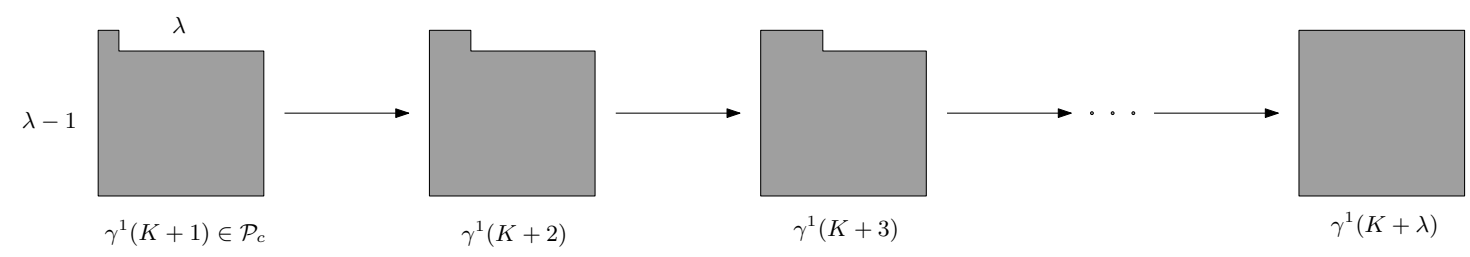

Figure 5: Construction of the flow when $\gamma(K+1)^{1} \in \mathcal{P}_{c}$

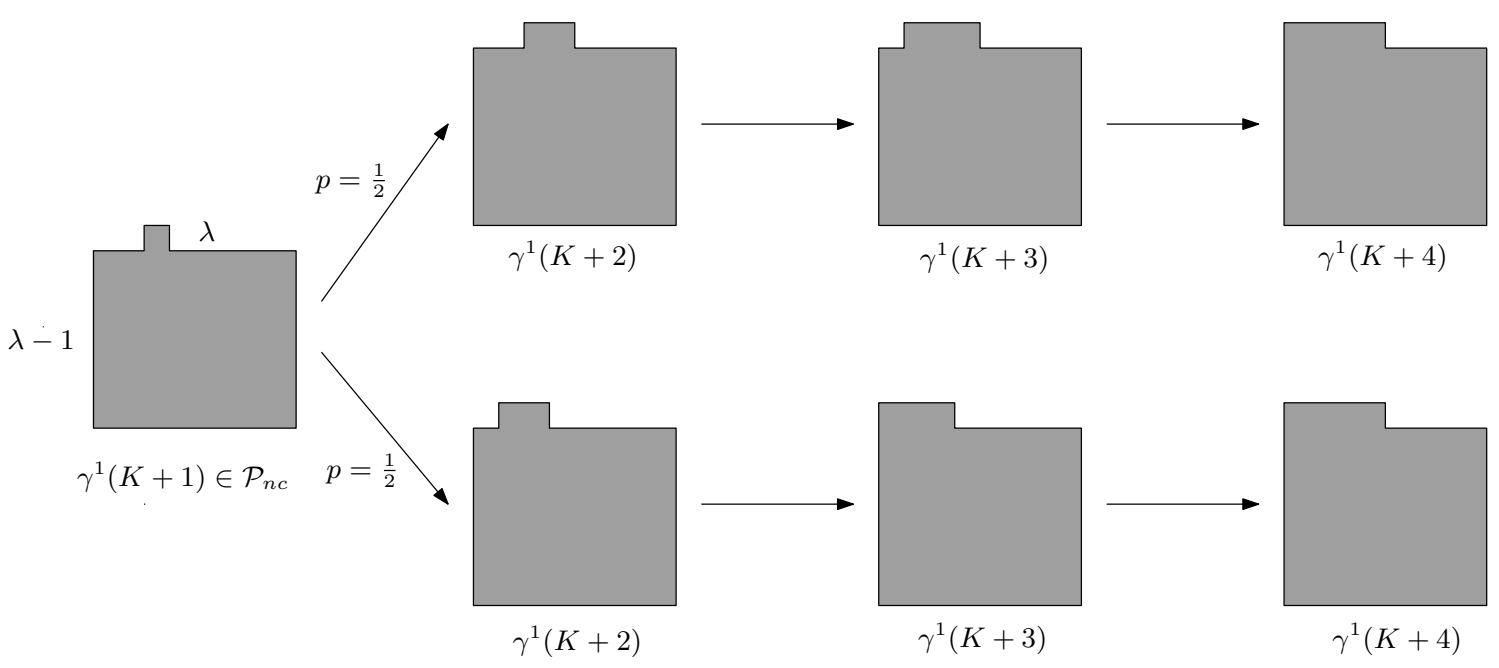

Figure 6: Construction of the flow when $\gamma^{1}(K+1) \in \mathcal{P}_{n c}$

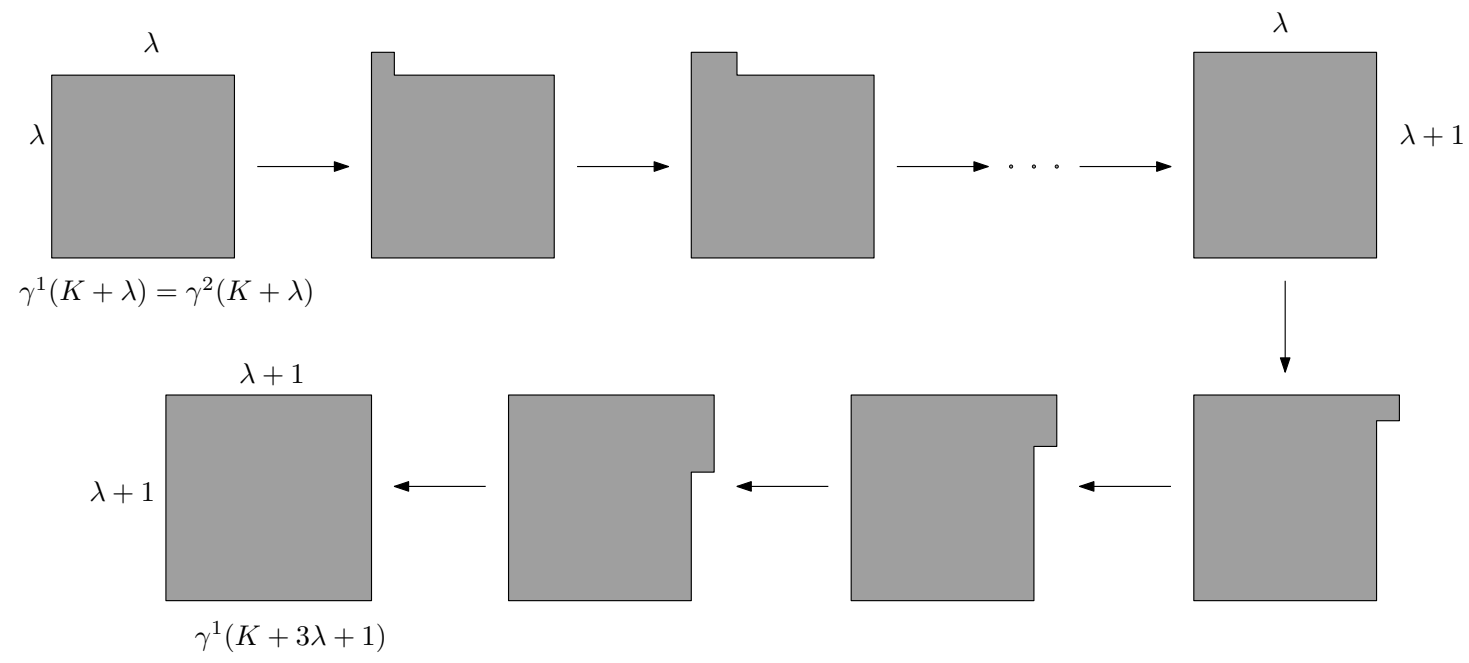

Figure 7: Construction of the supercritical flow 
Given a path $\gamma=(\gamma(0), \ldots, \gamma(|\Lambda|))$, with $\gamma(0)=-\underline{1}$ and $\gamma(|\Lambda|)=+\underline{1}$, we denote from now on with $\mathbb{P}(\gamma)$ the probability of the path $\gamma$ for the Markov chain associated with the flow $f$ :

$$
\mathbb{P}(\gamma):=\mathbb{P}^{f}(\mathbb{X}=\gamma)=\frac{\prod_{i=1}^{|\Lambda|} f(\gamma(i-1), \gamma(i))}{\prod_{i=1}^{|\Lambda|-1} F(\gamma(i-1))}
$$

Hence, the non-null probability paths from $-\underline{1} \rightarrow+\underline{1}$ of the Markov chain defined by the flow $f$ are of two types: we denote with $\mathcal{I}_{c}$ the set of the paths $\gamma$ such that $\gamma(K+1) \in \mathcal{P}_{c}$ and with $\mathcal{I}_{n c}$ the set of the paths such that $\gamma(K+1) \in \mathcal{P}_{n c}$. Thus, we have the following results: $\#\left\{\gamma \in \mathcal{I}_{c}\right\}=8|\Lambda|$ and that for $\gamma \in \mathcal{I}_{c}$

$$
\mathbb{P}(\gamma)=1 /(4|\Lambda| \lambda)=f(\gamma(K+1), \gamma(K+2))
$$

so that:

$$
\mathbb{P}\left(\mathcal{I}_{c}\right)=8|\Lambda| f\left(\gamma(K+1) \in \mathcal{P}_{c}, \gamma(K+2)\right)
$$

Analogously we have $\#\left\{\gamma \in \mathcal{I}_{n c}\right\}=8|\Lambda|(\lambda-2)$ and for $\gamma \in \mathcal{I}_{n c}$ :

$$
\begin{gathered}
\mathbb{P}(\gamma)=1 /(8|\Lambda| \lambda)=f(\gamma(K+1), \gamma(K+2)) \\
\mathbb{P}\left(\mathcal{I}_{n c}\right)=8|\Lambda|(\lambda-2) f\left(\gamma(K+1) \in \mathcal{P}_{n c}, \gamma(K+2)\right)
\end{gathered}
$$

By Proposition 5.1 and by the choice of the flow (6.48), we have

$$
\begin{aligned}
\operatorname{CAP}(-\underline{1},+\underline{1}) \geq & \sum_{\gamma \in \mathcal{I}_{n c} \cup \mathcal{I}_{c}} \mathbb{P}(\gamma)\left[\sum_{k=0}^{|\Lambda|-1} \frac{f(\gamma(k), \gamma(k+1))}{\mu_{\beta}(\gamma(k)) p(\gamma(k), \gamma(k+1))}\right]^{-1} \\
\geq & \mathbb{P}\left(\gamma \in \mathcal{I}_{c}\right) \frac{e^{-H(\gamma(K+1), \gamma(K+2))}}{Z f\left(\gamma(K+1) \in \mathcal{P}_{c}, \gamma(K+2)\right)}(1+o(1)) \\
& +\mathbb{P}\left(\gamma \in \mathcal{I}_{n c}\right) \frac{e^{-H(\gamma(K+1), \gamma(K+2))}}{Z f\left(\gamma(K+1) \mathcal{P}_{n c}, \gamma(K+2)\right)}(1+o(1))
\end{aligned}
$$

where in the last inequality we used Theorem 6.3 , since the paths are optimal paths for $\beta$ large enough. Hence, from the definition of the flow and from 2.12, we have:

$$
\begin{aligned}
\operatorname{CAP}(-\underline{1},+\underline{1}) \geq & \frac{\mu(-\underline{1}) e^{-\beta \Gamma}}{2^{|\Gamma|}}\left[\frac{\mathbb{P}\left(\gamma \in \mathcal{I}_{c}\right)}{f\left(\gamma(K+1) \in \mathcal{P}_{c}, \gamma(K+2)\right)}\right. \\
& \left.+\frac{\mathbb{P}\left(\gamma \in \mathcal{I}_{n c}\right)}{f\left(\gamma(K+1) \in \mathcal{P}_{n c}, \gamma(K+2)\right)}\right](1+o(1)) \\
\geq & \frac{\mu(-\underline{1}) e^{-\beta \Gamma}}{2^{|\Gamma|}}[8|\Lambda|+8|\Lambda|(\lambda-2)]=K_{1} \mu(-\underline{1}) e^{-\beta \Gamma}
\end{aligned}
$$




\section{References}

[BBI] A. Bianchi, A. Bovier, and D. Ioffe, Sharp asymptotics for metastability in the Random Field Curie-Weiss model. EJP 14, 1541-1603 (2009)

[BCLS] S. Bigelis, E.N.M. Cirillo, J.L. Lebowitz, E.R. Speer, Critical droplets in metastable probabilistic cellular automata. Phys. Rev. E 59, 3935 (1999).

[BEGK] A. Bovier, M. Eckhoff, V. Gayrard and M. Klein, Metastability and low lying spectra in reversible Markov chains. Commun. Math. Phys.\} 228, 219-255 (2002)

[BHN] A. Bovier, F. den Hollander and F.R. Nardi, Sharp asymptotics for Kawasaki dynamics on a finite box with open boundary. Probab. Theory Relat. Fields 135, 265-310 (2006)

[BHS] A. Bovier, F. den Hollander and C. Spitoni, Homogeneous nucleation for Glauber and Kawasaki dynamics in large volumes and low temperature. Ann. Probab. 38, 661-713 (2010)

[BK] K. A. Berman, M. H. Konsowa, Random paths and cuts, electrical networks, and reversible Markov chains, SIAM J. Discrete Math. 3, 311-319 (1990)

[BM] A. Bovier and F. Manzo, Metastability in Glauber dynamics in the low-temperature limit: beyond exponential asymptotics. J. Stat. Phys. 107, 757-779 (2002)

[BNS] A. Bovier, F.R. Nardi, C. Spitoni, Sharp Asymptotics for Stochastic Dynamics with Parallel Updating Rule with self-interaction EURANDOM Report 2011-08

[Bo] A. Bovier, Metastability: a potential theoretic approach. Proceedings of ICM 2006, EMS Publishing House, pp. 499-518. (2006)

[CGOV] M. Cassandro, A. Galves, E. Olivieri, M.E. Vares, Metastable behavior of stochastic dynamics: A pathwise approach. Journ. Stat. Phys. 35, 603-634 (1984)

[CN] E.N.M. Cirillo, F.R. Nardi, Metastability for the Ising model with a parallel dynamics. Journ. Stat. Phys. 110, 183-217 (2003)

[CNS] E.N.M. Cirillo, F.R. Nardi, C. Spitoni, Metastability for reversible probabilistic cellular automata with self-interaction. Journ. Stat. Phys. 132, 431-471 (2008)

[CNS2] E.N.M. Cirillo, F.R. Nardi, C. Spitoni, Competitive nucleation in reversible probabilistic cellular automata. Phys. Rev. E. 78, 040601 (2008)

[De] B. Derrida, Dynamical phase transition in spin models and automata. In: van Beijeren, H. (ed.) Fundamental problems in Statistical Mechanics, vol. VII. Elsevier (1990) 
[Ho] F. den Hollander, Three lectures on metastability under stochastic dynamics. In Methods of Contemporary Mathematical Statistical Physics. Lecture Notes in Math. 1970. Springer, Berlin (2009)

[MNOS] F. Manzo, F.R. Nardi, E. Olivieri, E. Scoppola, On the essential features of metastability: tunneling time and critical configurations. Journ. Stat. Phys. 115, 591-642 (2004)

[OS] E. Olivieri, E. Scoppola, Markov chains with exponentially small transition probabilities: First exit problem from a general domain. I. The reversible case. J. Stat. Phys. 79, 613647 (1995)

[OS2] E. Olivieri, E. Scoppola, Markov chains with exponentially small transition probabilities: First exit problem from a general domain. II. The general case. J. Stat. Phys. 84, 987-1041 (1996)

[OV] E. Olivieri, M.E. Vares, Large deviations and metastability. Cambridge University Press, Cambridge, UK, (2004) 